\title{
CONCEPTUAL DESIGN FOR ADDITIVE MANUFACTURING: AN EXPLORATIVE STUDY
}

\author{
F. Valjak ${ }^{1, \otimes}$, N. Bojčetić ${ }^{1}$, A. Nordin ${ }^{2}$ and D. Godec ${ }^{1}$ \\ ${ }^{1}$ University of Zagreb, Croatia, ${ }^{2}$ Lund University, Sweden \\ $\square$ filip.valjak@fsb.hr
}

\section{Abstract}

With the broader industrial application of Additive Manufacturing (AM), designers are facing new challenges in conceptual design for AM. To better understand the problematic, the authors organised a design workshop with six experts in AM. The paper presents the results of the conducted design workshop and discusses the current and future trends in research on the conceptual design for AM.

Keywords: conceptual design, additive manufacturing, design practice

\section{Introduction}

The conceptual design phase has a unique role in the overall design process. In the conceptual phase, the overall layout of the product is established, and decisions made here have a vast influence on following phases and final design of the product (Pahl et al., 2007). Furthermore, the structured design phases and the overall process have a positive influence on the design of innovative products (Segonds et al., 2016). One of the most thought methods for conceptual design to be used in the structured and prescribed design process is the functional decomposition (Tomiyama et al., 2009). Functional decomposition is used for decomposition of the overall function of the product into its subfunction to ease the search of partial solutions. And while the method is widely used in academia, due to its drawbacks in performing routine design or improvement of design improvement it is less utilised in the industrial practice (Fiorineschi et al., 2016; Tomiyama et al., 2009).

On the other hand, Additive Manufacturing (AM) brought new design freedom and design possibilities (Gibson et al., 2015) that can be utilised to achieve innovation in products. At the same time, the advent of AM in recent years changed the way how we manufacture our products but also changed the product we make and consequently this requires us to change the way how we design our product (Seepersad, 2014). Therefore, a new design approach emerged - Design for Additive Manufacturing (DfAM) that is focused on the utilisation of unique possibilities of AM. The recent surveys showed the increasing application of DfAM methods and tools in professional design practice (Pradel et al., 2018). Currently, the designers use DfAM mostly in embodiment and detail design phases, but at the same time acknowledge the need to apply it earlier in the design process (Pradel et al., 2018) to utilise its full potential.

Therefore, this paper investigates the current design practice in the conceptual phase of the AM oriented design process, as well as the current state of the DfAM literature on conceptual design. Furthermore, the use of a structured design approach according to Pahl \& Beitz model of the design 
process is investigated. Here the focus was put on the use of a functional model of a product during the conceptual design of AM product. The conducted research was built around two research questions:

\section{RQ1: What is the current design practice in conceptual DfAM process? \\ RQ2: Could the use of a functional model be beneficial for conceptual DfAM process and provide guidance in the conceptual phase?}

To answer the research questions, the design workshop "Conceptual Design for AM" was organised with six experts in AM and DfAM.

The paper is organised as follows in Section 2 current state of the art in conceptual DfAM is presented. In Section 3 method of the research and protocol of the conducted design workshop is described with results presented in Section 4. Section 5 discusses the results and observations from the workshop, while Section 6 gives the overall conclusion of the conducted research.

\section{Background and related work}

The DfAM as a design approach is a topic of research for the last two decades. The research focus of first DfAM approaches was mostly on the embodiment and detail design, while the conceptual design phase was often neglected until recently. The first broader research on how AM knowledge can be stored and applied in conceptual design was conducted by Bin Maidin et al. (2012). They captured comprehensive AM knowledge in the form of AM design features stored in an MS Access database. The designers search the database by answering questions that guide them to appropriate AM design feature for their design problem.

Similarly, Blösch-Paidosh and Shea (2017) reviewed over 200 AM products to capture and store AM design knowledge in the form of Design Heuristics for AM. The Design Heuristics capture broad AM knowledge on a high level of abstraction suitable for application in the early phases of the design process and conceptualisation of AM products. The conducted studies and experiments with students and professional designers (Blösch-Paidosh and Shea, 2019; Lindwall and Törlind, 2018) showed the positive influence of heuristics on concept creation during ideation sessions.

On the other hand, various authors investigate the Design Principles as a source of AM design knowledge. Weiss et al. (2016) proposed the use of AM Design Principles in the form of design catalogue where the design principles are organised as partial solutions for the variety of functions. Similarly, Watschke et al. (2019) and Valjak and Bojčetić (2019) proposed the use of design principles in the form of a computer database. Both approaches also use functions for organising design principles, but while Watschke et al. focus only on capturing AM knowledge about a multimaterial aspect of AM, Valjak \& Bojčetić propose the establishment of a repository with comprehensive AM knowledge.

Beside research regarding formalising and storing AM design knowledge that can be used in the conceptual design phase, it is important to acknowledge the research on methods for conceptual AM design as well. The main purpose of the hitherto developed methods for AM conceptual design is to stimulate divergent thinking and support exploration of the design space enabled through uniqueness and possibilities of AM. Laverne et al. (2015) proposed the method for early AM assembly design that consists of three stages in conceptual design used for generation of concepts, working principles and working structures. Similarly, Rias et al. (2017) developed a five-step approach for enhancing creativity in AM orientated conceptual design. The specificity of their method is the combination of divergent and convergent steps where firstly AM design space is explored and gathered ideas are assessed before AM concepts are created and evaluated.

Furthermore, few authors focused their research on function-based methods for conceptual AM design. Boyard et al. (2013) proposed the use of the 3D graph of functions that can be spatially reorganised to determine the architecture of the product. Comparison of the created graph with existing graphs from the database of AM solutions can enable reconfiguration of the product layout regarding the specificities of AM. On the other hand, Valjak et al. (2018) proposed the method based on the functional model of a product. In their approach, a functional model of a product is mapped with the design principles for AM from which the morphological matrix is created. The morphological 
matrix provides different AM based partial solutions for product functions or block of functions. Through various combinations of AM partial solution, one or more working structures and concepts are created that utilise unique possibilities of AM. Similarly, Borgue et al. (2018) proposed the method based on Enhanced Function-Means modelling (EF-M). Their method is used for the redesign of existing components for AM, but besides AM potentials also consider the current limitations of AM technologies.

\section{Method}

To investigate the stated research questions and gain a better understanding of AM oriented conceptual design phase authors organised a small design workshop with six AM experts from academia. The participants are from two European universities and have been working with AM technologies on average between 3 and 5 years, while one participant had 25 years of experience with AM. Furthermore, participants have worked with both polymer and metal materials on various types of AM technologies (e.g. material jetting, material extrusion, powder-bed fusion). Four participants have had industrial experience as product developers before starting their academic careers, and still have connections with the industrial stakeholders thorough consultancy about AM, tasks of the redesign for AM and creation of own designs. All participants are familiar with one or more models of the design process (e.g. Pahl \& Beitz model), as well with the concept of the functional model and its application in conceptual design.

The design workshop consisted of five steps, with a total duration of approximately 85 minutes (Figure 1). At the beginning of the workshop, the participants were informed about the objectives and purpose of the workshop, protocol and the design task. In the design task activity, participants were randomly divided into two groups, with three participants in each group. Both groups had the same design task. The design task was to create an AM concept of a citrus press (citrus reamer) powered only with human energy. The citrus press was chosen as it is a simple product regarding functions, with a potentially complex geometrical shape. Furthermore, the participants were familiar with the product, and the time need for conceptualisation of the citrus press was suitable for the available slot. The description of the product was read aloud to the participants before the beginning of the design task.

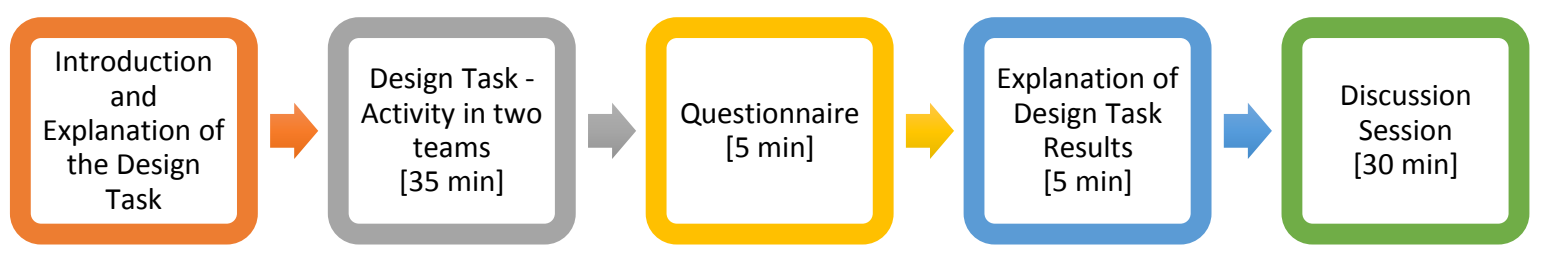

Figure 1. Design workshop protocol

Furthermore, the participants were given the functional model of the citrus press (Figure 2). The functional model consisted of functions and flows according to Hirtz's function basis vocabulary (Hirtz et al., 2002). The use of predefined vocabulary ensured the high level of abstraction, which do not suggest technical solutions, needed in the functional model. To ensure the participants have good understanding of functions and flows, definitions of used functions and flows were provided. The functional model was given to the participants as they were not familiar with the predefined vocabulary and the available time slot was not suitable for additional explanation of vocabulary. Hence, the participants create the functional model themselves. Therefore, the participants were instructed to analyse the functional model and use it to generate the concept. Furthermore, they were told to be open-minded, to explore all possibilities of AM they are familiar with and to neglect the limitations of AM. They were asked to mark how did they solve a particular function or block of functions on their final concept. The design task started with the functional model as it is an abstract description of the product that do not suggest the technical solution. The participants were instructed to find as many AM solutions for each function but were not asked to create a formal morphological matrix. 


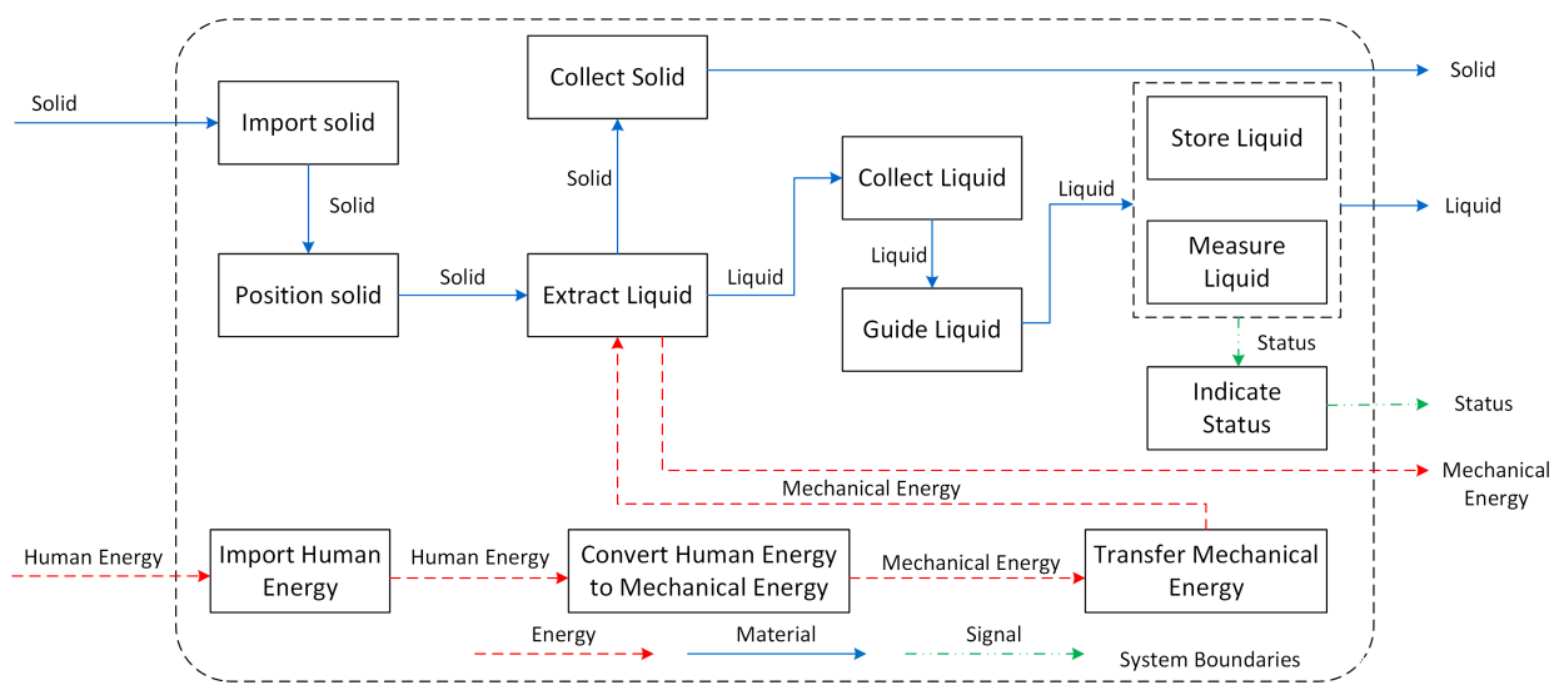

Figure 2. Function model of the citrus press

The design task lasted 35 minutes, and participants were given notice 10 minutes before the end to finish up their final concepts. The activity was recorded with two video cameras, each recording one group.

After the design task, participants were asked to fill up the questionnaire with ten questions, three regarding the current state of conceptual DfAM, and seven regarding conducted task and use of the functional model. The questionnaire was asked right after the design task to avoid the influence of the discussion on answers of the participants (Coolican, 2017).

In the final part of the workshop, both groups firstly presented their concepts and explained which AM features were utilised in the concepts. Afterwards, an open discussion session started. The discussion was moderated by one researcher, who opened the various topics for discussion and asked the questions and subquestions. The topics in the discussion were: the conducted design task, the current state of the conceptual DfAM, personal experiences with conceptual DfAM, and future of AM. The discussion session lasted for approximately 30 minutes. The discussion session was recorded with two video cameras.

\section{Results}

\subsection{The design task}

The concepts created in the design activity of the workshop incorporate unique AM features to solve the given design problem. The first concept is based on the principle of using a lever to convert human energy into mechanical energy (Figure 3). The central part of the device for positioning and extracting juice from citrus, utilise the geometrical complexity enabled with AM. It consists of several small holes with matching spikes to ensure satisfying extraction of juice. Furthermore, structures in the inside of the central hub are used for filtering juice from pulp and seeds. Both the inside structures and outside shape guide the juice to the bottom where it drops into a bowl.

The second concept (Figure 4) uses rotation to extract the juice from the citrus. The citrus is placed on the top of the spiral-shaped central part. The user holds the citrus with one hand and rotates the handle with the other. Rotation from the handle is transferred on the central part through integrated gear. The concept utilises possibilities of AM to integrate several functions and shapes in the single component. The central part has a spiral in the middle for juice extraction, with the small perforation for filtering the juice. The juice is collected with the concave bottom and guided into a bowl underneath. The central part has integrated gear at the outer rim.

Both concepts integrate multiple functions in a single part and are focused on the complexity of geometry. Nevertheless, it is important to notice that the created designs are achievable with other manufacturing technologies as well, but the AM reduce the number of manufacturing operations and parts in a product. 


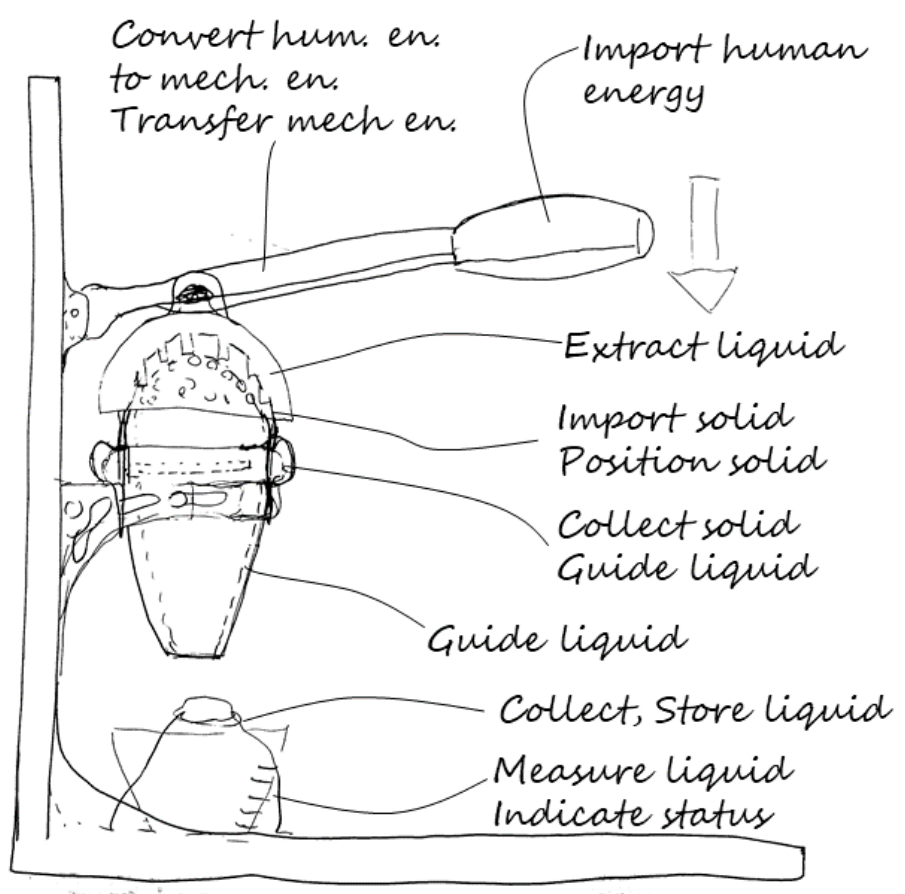

Figure 3. Concept one created in the design task

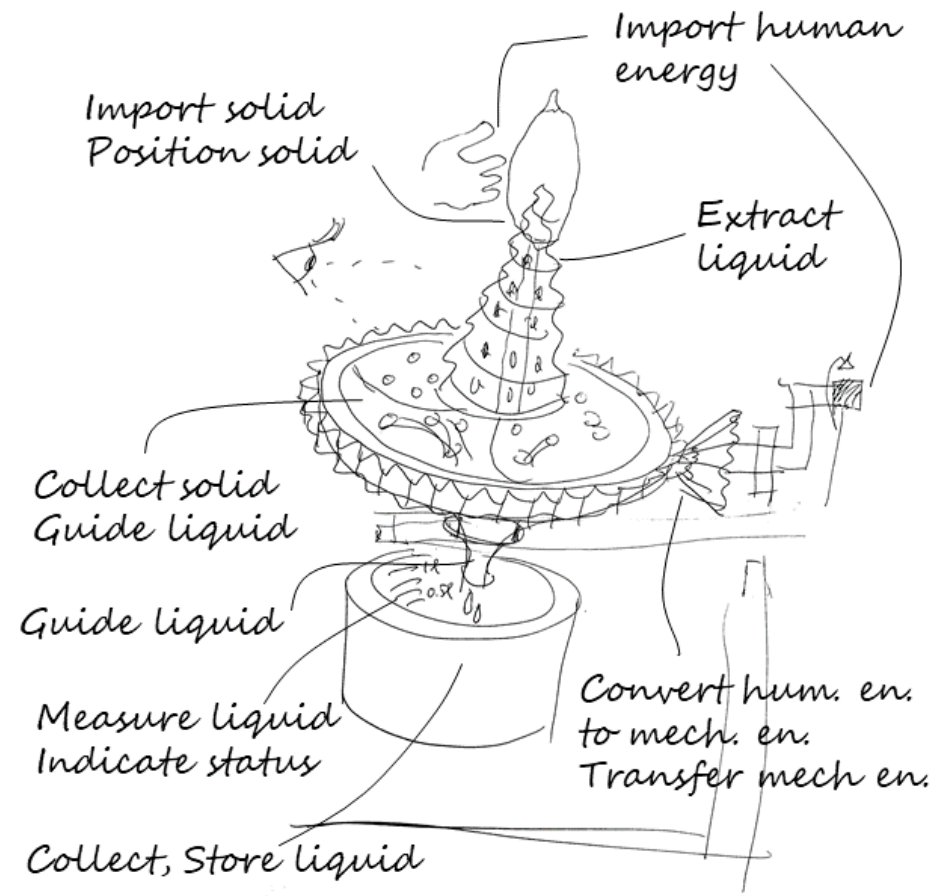

Figure 4. Concept two created in the design task

After the conducted design task, the participants were given a questioner with ten questions. The results of the questioner can be seen in Table 1. First three questions asked the participants about the current state of the art inside the DfAM. Two participants refrained from answering as they are not familiar with the latest literature on DfAM. As one of the objectives of the design task was to investigate the use of the functional model in the conceptual design phase, the next three questions were about the used functional model. From the result, it can be noticed that participants did not have a problem with the understanding of the given functional model and found it as a useful aid in the given design task. 
Table 1. Results of the conducted questioner

\begin{tabular}{|c|c|c|c|c|c|}
\hline Statement/Number of answers & 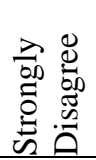 & 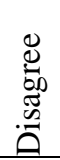 & 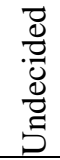 & $\underset{\mathscr{0}}{\stackrel{0}{L}}$ & 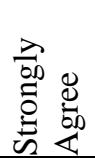 \\
\hline $\begin{array}{l}\text { 1. Existing methods and tools for conceptual design (e.g. Disney method, } \\
\text { Brainstorming, Morphological matrix) are good enough for conceptual AM } \\
\text { design, and there is no need for specialised AM conceptual design support. }\end{array}$ & & 3 & & & 1 \\
\hline $\begin{array}{l}\text { 2. The hitherto developed DfAM methods and tools for conceptual AM } \\
\text { design are applicable in the design practice. }\end{array}$ & & & & 4 & \\
\hline $\begin{array}{l}\text { 3. I often use the DfAM methods \& tools for conceptual AM design in } \\
\text { everyday design practice. }\end{array}$ & & 1 & 1 & 1 & 1 \\
\hline 4. The functional model was clear and understandable. & & & & 2 & 4 \\
\hline $\begin{array}{l}\text { 5. The provided definitions of functions and flows were easy to understand } \\
\text { and comprehend. }\end{array}$ & & & & 3 & 3 \\
\hline $\begin{array}{l}\text { 6. The functional model was on a high-level of abstraction (it did } \\
\text { not suggest the technical solutions). }\end{array}$ & & & & 4 & 2 \\
\hline 7. The functional model did not have enough functions. & 2 & 3 & & & 1 \\
\hline 8. The functional model did not have enough flows. & 2 & 2 & & 1 & 1 \\
\hline $\begin{array}{l}\text { 9. The use of a functional model was helpful during the conceptualisation of } \\
\text { the given product. }\end{array}$ & & & & 4 & 2 \\
\hline 10. The function-based methods are useful tool in conceptual AM design. & & & & 5 & 1 \\
\hline
\end{tabular}

\subsection{The discussion session}

The open discussion started with questions regarding the conducted design task and functional model, continued with general questions regarding conceptual DfAM and challenges participants are facing in everyday design practice, sources of AM knowledge, as well as the application of DfAM in industry. The summary of the discussed topics, problems designers are facing, and challenges of DfAM are described in this section.

The general consensus among the participants is that a functional model is a useful tool in the context of AM conceptual design, as it guides through the design activities in the conceptual phase. They mentioned that they used the functional model only at the beginning of the design task and for the final check, as the product was quite simple, but acknowledge its usefulness when the product is more complex with a larger number of functions and connected flows.

On the topic of conceptual AM design in their design practice, participants mentioned that the majority of the parts they design are redesigns of the conventional parts for AM. This is due to cooperation with the industry that often asks for redesigns for AM rather than entirely new designs. On the other hand, completely new designs for AM often emerge in research projects. The biggest challenge they are facing in such new AM part designs is the lack of knowledge and experience regarding particular design feature or parameter. Therefore, they often do a lot of testing and try-outs to find the boundaries and constraints of the design space. The process is time-consuming, and the design phases (conceptual, embodiment and detail design phase) are interrelated, often done in parallel and in few iterations without clear boundaries between phases, until a satisfying design is found. The latter is related to the available sources of AM knowledge. The participants mentioned they mostly rely on their previous experience as the lack of sources on AM knowledge is problematic.

Furthermore, the participants are facing difficulties regarding the types of available sources of AM knowledge. The literature participants are using is deep technical literature (one participant gave the example of "paper on crack propagation through the titanium printed part"). Additionally, 
participants mentioned the lack of literature on DfAM, according to participant: "only a few websites with basic guidelines and not a comprehensive source of knowledge". Additionally, the problem of educating students emerged in the discussion. One participant said:
“...It's important for students to learn how to think [regarding design for additive manufacturing], wall thickness and hole sizes you can look up and google, but it is thought process behind, how to approach additive that is missing..."

The final topic of the session was about the application of DfAM in industry. According to the experience of the participant most companies are using exiting designs, with small changes, they then want to print, which is often very expensive. Very few companies are designing and redesigning their products to suit the additive manufacturing. When asked why the companies are not using AM more and why they have problems in adopting their designs to suit additive, one participant said:

\section{“...I don't think the companies have the knowledge, they all have heard about additive manufacturing, and they think it's instant production, and perfect parts coming off beautifully, and then they come to us..."}

Furthermore, the companies have the established design process, and are reluctant to changes:

$$
\begin{aligned}
& \text { "...Hundreds of companies are still saying part must be made of } 316 \text { stainless steel. } \\
& \text { And you ask why, and the answer is "that's the way we always made it”. So, they } \\
& \text { don't think what's the function of the part, which is where this [functional model] } \\
& \text { comes in, and could titanium or aluminium or some other material, do it better and } \\
& \text { cheaper because you use it less of it. But a lot of it is historical, it's designed for } \\
& \text { certain material, for history reasons rather for real practical..." }
\end{aligned}
$$

Besides the above problems, participants mentioned the lack of qualification process for AM parts. Many industries, such as aerospace and medical, have rigours regulations for the use of critical components. Due to the novelty of AM, properties of AM parts, in the long run, are still relatively unknown.

In the end, the discussion raised some open questions and problems regarding DfAM and provided valuable insight into the current state of DfAM.

\section{Discussion}

The conducted design workshop, although limited in its scope and number of participants, as well as their profile, provided limited but valuable insight into conceptual design for AM. The design task resulted in two different concepts of the citrus press. And while the participants were instructed to explore the entire design space enabled by AM, they mostly focused only on the geometrical complexity enabled by AM. This can be contributed to two factors, simplicity of the given design task, and previous experience of participants who usually use only geometrical freedom of AM in their everyday design activities. Furthermore, experienced designers often have a problem of thinking out of their traditional mindset (Seepersad, 2014). This can be seen in the created concepts, where the holders and cups do not utilise AM and are better suited for conventional manufacturing technologies. Based on the results of the design task and follow up discussion it can be concluded there is a need for the development of design tools and methods that will enable exploration of the AM design space and remove the cognitive barriers in conceptual AM design. Use of functional model for AM conceptual design can be one of the ways how to approach this problem. The conducted design task showed some benefits of the approach (e.g. common understanding of the design problem (Little and Wood, 1997)), but also the drawbacks as it is not suitable for all design problems. Consequently, other design approaches, not included in this study, must be reviewed in the context of AM as well.

Another problem identified by the participants is the lack of sources of AM knowledge. As the area of DfAM is a relatively new field of research, the boundaries of the AM design space and limits of the technology are still being investigated. Furthermore, the current sources are often about the detail design and provide only guidelines regarding parameters of the design features (e.g. rules for SLS, Seepersad et al. (2012)). There is a growing need to think additively (Pradel et al., 2018) and change the paradigm of 
how to approach the AM oriented design process (Seepersad, 2014). To support the stated, new comprehensive sources of AM knowledge are needed that will provide guidance on how to design for AM (e.g. Diegel et al., (2019)). The sources themselves will not be sufficient, and education of future engineers who will work with AM and design products for AM is also needed (Watschke et al., 2017).

The challenges described above are also connected with the current status of DfAM in industry. The problematic regarding the lack of DfAM knowledge in the industry was already identified in a comprehensive study conducted by Pradel et al. (2018). The participants of the workshop mentioned they encountered similar problems in their work with industrial partners. Furthermore, they identified the problem of using paradigm "that's how we always did" as a major issue in adopting the DfAM methodologies in the industry. Consequently, designers do not adopt their design to suit AM and potentials and benefits of AM are not utilised to full potential. It is important to emphasise that some problems participants mentioned, such as the latter, are not exclusively related to the DfAM but rather to conceptual design in general, independent of the used technology for the manufacturing of the final product.

The AM opened new design space that should be explored during the conceptual design stage of the design process. The possibility of AM to create (almost) any shape imaginable, together with other advantages of AM such as multi-material fabrication, (Gibson et al., 2015), are examples of advance possibilities of AM that enable new possibilities in design that were hardly possible before using conventional manufacturing technologies. During the conceptual design, designers need to be encouraged to think about the new possibilities and new ideas to create new products and utilise the AM design space.

There are a few limitations of the conducted study and design workshop for broader conclusions regarding conceptual DfAM. Firstly, the design workshop participated only six experts, but more importantly, all of them are from academia with some prior experience in industrial design practice. The lack of industrial design practitioners is a major drawback as they could be experiencing different challenges in the everyday AM oriented design practice. Furthermore, due to the limited time slot available, the design task and related functional model were quite simple. The participants studied the functional model on the begging of the design task and used it rarely during the concept generation but rather just referred to it at the end of the task for a final check-up. The use of a functional model with more functions, or product that the participants are not familiar with, could increase the use of functional model throughout the design task. Such activity could provide new insight into the use of a functional model for the AM conceptual design. Furthermore, the functional model was developed by the researchers conducting the experiment, and as such, was given to the participants. If the participants had an opportunity to create their own functional model of a given product, it could be speculated that they would spend more time using the functional model during conceptualisation and would have referenced it more often, and the used functions would be different. The stated was not included in the design workshop due to limited time available. Nevertheless, authors are planning to conduct a proper design experiment with a controlled environment, where the participants would develop their own functional models to be used in the conceptualisation design task. Afterwards, a rigours analysis of gathered data, such as protocol analysis, could be conducted to investigate the dynamic and the ways how the functional model is used throughout the design task.

\section{Conclusion}

This study explores the current status of DfAM with focus on conceptual design and use of a functional model in the conceptual AM design. To investigate the research questions, a design workshop with six experts on AM from academia was organised. The workshop consisted of two activities, design task to investigate the use of functional model and discussion session to gain an overall insight into conceptual DfAM. The results of the workshop indicate the current challenges designers are facing when designing components for AM. The most noticeable is the lack of comprehensive sources of AM knowledge and problems of how to think and design additively. Furthermore, the conducted design task showed the potentials of using a functional model in the context of conceptual AM design. Due to limitations of the conducted workshop, further research is planned to gain additional understanding of the conceptual AM. 


\section{Acknowledgement}

This paper is part of the research included in project Increasing Excellence on Advanced Additive Manufacturing - H2020 (G.A. 810708), financed by the European Union. The authors would like to thank the EU for the financing of the project.

\section{References}

Bin Maidin, S., Campbell, I. and Pei, E. (2012), "Development of a design feature database to support design for additive manufacturing”, Assembly Automation, Vol. 32 No. 3, pp. 235-244. https://doi.org/10.1108/ 01445151211244375

Blösch-Paidosh, A. and Shea, K. (2017), "Design heuristics for additive manufacturing”, DS 87-5 Proceedings of the 21st International Conference on Engineering Design (ICED 17) Vol 5: Design for X, Design to X, Vancouver, Canada, August 21-25, 2017, The Design Society, Vancuver, Canada, pp. 091-100.

Blösch-Paidosh, A. and Shea, K. (2019), "Design Heuristics for Additive Manufacturing Validated Through a User Study”, Journal of Mechanical Design, Vol. 141 No. 4, p. 041101. https://doi.org/10.1115/1.4041051

Borgue, O. et al. (2018), "Function modelling and constraints replacement to support design for additive manufacturing of satellite components”, DS 91: Proceedings of NordDesign 2018, Linköping, Sweden, August 14-17, 2018, The Design Society, Linköping, pp. 1-10.

Boyard, N. et al. (2013), “A design methodology for parts using additive manufacturing”, 6th International Conference on Advanced Research in Virtual and Rapid Prototyping, pp. 399-404.

Coolican, H. (2017), Research Methods and Statistics in Psychology, Psychology Press, London. https://doi.org/10.4324/9780203769836

Diegel, O., Nordin, A. and Motte, D. (2019), A Practical Guide to Design for Additive Manufacturing, Springer Singapore, Singapore, https://doi.org/10.1007/978-981-13-8281-9

Fiorineschi, L., Rotini, F. and Rissone, P. (2016), "A new conceptual design approach for overcoming the flaws of functional decomposition and morphology”, Journal of Engineering Design, Vol. 27 No. 7, pp. 438-468. https://doi.org/10.1080/09544828.2016.1160275

Gibson, I., Rosen, D. and Stucker, B. (2015), Additive Manufacturing Technologies, Springer New York, New York, NY. https://doi.org/10.1007/978-1-4939-2113-3

Hirtz, J. et al. (2002), "A functional basis for engineering design: Reconciling and evolving previous efforts", Research in Engineering Design, Vol. 13 No. 2, pp. 65-82. https://doi.org/10.1007/s00163-001-0008-3

Laverne, F. et al. (2015), "Assembly Based Methods to Support Product Innovation in Design for Additive Manufacturing: An Exploratory Case Study", Journal of Mechanical Design, Vol. 137 No. 12. https://doi.org/10.1115/1.4031589

Lindwall, A. and Törlind, P. (2018), "Evaluating design heuristics for additive manufacturing as an explorative workshop method", DS 92: Proceedings of the DESIGN 2018 15th International Design Conference, The Design Society, Dubrovnik, Croatia, pp. 1221-1232. https://doi.org/10.21278/idc.2018.0310

Little, A.D. and Wood, K.L. (1997), "Functional Analysis: A Fundamental Empirical Study for Reverse Engineering, Benchmarking, and Redesign", ASME DTM Conference, pp. 1-21.

Pahl, G. et al. (2007), Engineering Design, Springer London, London, https://doi.org/10.1007/978-1-84628-319-2

Pradel, P. et al. (2018), "Investigation of design for additive manufacturing in professional design practice", Journal of Engineering Design, Vol. 29 No. 4-5, pp. 165-200. https://doi.org/10.1080/09544828.2018.1454589

Rias, A.-L. et al. (2017), "Towards additive manufacturing of intermediate objects (AMIO) for concepts generation", International Journal on Interactive Design and Manufacturing (IJIDeM), Vol. 11 No. 2, pp. 301-315. https://doi.org/10.1007/s12008-017-0369-0

Seepersad, C.C. (2014), "Challenges and Opportunities in Design for Additive Manufacturing", 3D Printing and Additive Manufacturing, Vol. 1 No. 1, pp. 10-13. https://doi.org/10.1089/3dp.2013.0006

Seepersad, C.C. et al. (2012), “A designer's guide for dimensioning and tolerancing SLS parts”, 23rd Annual International Solid Freeform Fabrication Symposium - An Additive Manufacturing Conference, SFF 2012; Austin, TX; United States; August 6-8, 2012, pp. 921-931.

Segonds, F. et al. (2016), "PLM and early stages collaboration in interactive design, a case study in the glass industry”, International Journal on Interactive Design and Manufacturing (IJIDeM), Vol. 10 No. 2, pp. 95-104. https://10.1007/s12008-014-0217-4

Tomiyama, T. et al. (2009), "Design methodologies: Industrial and educational applications", CIRP Annals, Vol. 58 No. 2, pp. 543-565. https://10.1016/j.cirp.2009.09.003

Valjak, F. and Bojčetić, N. (2019), "Conception of Design Principles for Additive Manufacturing”, Proceedings of the Design Society: International Conference on Engineering Design, Vol. 1 No. 1, pp. 689-698. https://10.1017/dsi.2019.73 
Valjak, F., Bojčetić, N. and Lukić, M. (2018), "Design for Additive Manufacturing: Mapping of product functions", DS 92: Proceedings of the DESIGN 2018 15th International Design Conference, The Design Society, Dubrovnik, Croatia, pp. 1369-1380. https://10.21278/idc.2018.0364

Watschke, H. et al. (2017), "A methodical approach to support ideation for additive manufacturing in design education", DS 87-5 Proceedings of the 21st International Conference on Engineering Design (ICED 17) Vol 5: Design for X, Design to X, Vancouver, Canada, August 21-25, 2017, The Design Society, pp. 41-50.

Watschke, H. et al. (2019), “A Methodical Approach to Support Conceptual Design for Multi-Material Additive Manufacturing”, Proceedings of the Design Society: International Conference on Engineering Design, Vol. 1 No. 1, pp. 659-668. https://10.1017/dsi.2019.70

Weiss, F., Binz, H. and Roth, D. (2016), "Conception of a design catalogue for the development of functionalities with additive manufacturing", DS 85-2: Proceedings of NordDesign 2016, Volume 2, Trondheim, Norway, August 10-12, 2016, The Design Society, Trondheim, pp. 002-011. 